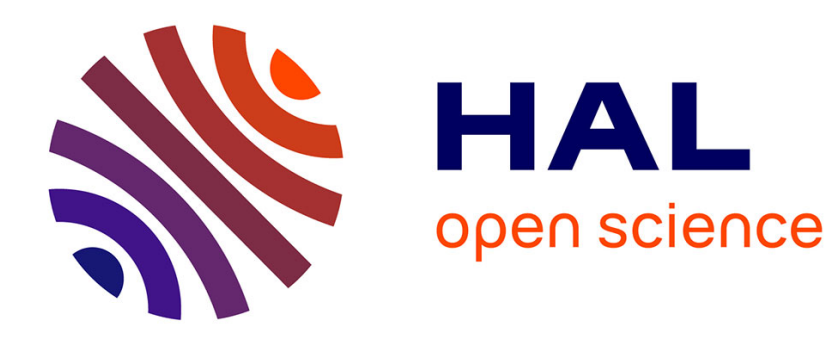

\title{
Le ferromagnétisme et l'état métallique \\ Louis Néel
}

\section{To cite this version:}

Louis Néel. Le ferromagnétisme et l'état métallique. J. Phys. Radium, 1940, 1 (7), pp.242-250. 10.1051/jphysrad:0194000107024200 . jpa-00233750

\section{HAL Id: jpa-00233750 https://hal.science/jpa-00233750}

Submitted on 1 Jan 1940

HAL is a multi-disciplinary open access archive for the deposit and dissemination of scientific research documents, whether they are published or not. The documents may come from teaching and research institutions in France or abroad, or from public or private research centers.
L'archive ouverte pluridisciplinaire HAL, est destinée au dépôt et à la diffusion de documents scientifiques de niveau recherche, publiés ou non, émanant des établissements d'enseignement et de recherche français ou étrangers, des laboratoires publics ou privés. 


\title{
LE FERROMAGNÉTISME ET L'ÉTAT MÉTALLIQUE
}

\author{
Par Louis NÉEL. \\ Conférence faite devant la Société française de Physique, le i5 avril ig39, à I 7 h. \\ Sommaire. - On se propose, dans cette conférence, d'exposer quelques-uns des résultats des \\ recherches entreprises à Strasbourg depuis dix ans sur le moment magnétique et le champ moléculaire \\ des métaux. Il s'agit, avant tout, d'une vue d'ensemble et un certain nombre de points délicats seront \\ intentionnellement laissés de côté.
}

Structure électronique et moment magnétique atomique. - Parmi les métaux, un certain nombre possèdent des propriétés magnétiques spécialement intéressantes : ce sont ceux qui, dans la classification périodique, ont reçu le nom d'éléments de transition. Je m'attacherai plus particulièrement à la première série de transition et dans celle-ci aux éléments qui la terminent : chrome, manganèse, fer, cobalt et nickel.

En commençant par les couches les plus profondes, la structure électronique de ces éléments est la suivante : des couches complètes semblables à celles du néon et faiblement diamagnétiques, puis un étage incomplet $3 d$ situé à l'intérieur d'un autre étage également incomplet, l'étage $4 s$. L'étage $3 d$ étant incomplet possède un moment cinétique résultant et corrélativement un moment magnétique. Cet étage, relativement profond, est assez bien protégé contre les actions extérieures; il joue, dans les propriété chimiques, un rôle moins important que l'étage $4 \mathrm{~s}$. Dans les sels, comme $\mathrm{NiCl}^{2}$, les électrons $4 s$, électrons de valence, sont partis tandis que l'étage $3 d$ est resté incomplet. Ce sel est paramagnétique et l'aimantation $I$, fonction de la température $T$ et du champ magnétique $H$, suit approximativement la loi de Curie

$$
\mathfrak{J}=\frac{C_{0} H}{T} ;
$$

le champ aligne les moments atomiques, mais cette action directrice est contre-balancée par l'agitation thermique. $C_{0}$ est une constante - constante de Curie - liée au moment atomique, dans les sels en question, par des formules compliquées. D'une part, en effet, le moment atomique est la résultante d'un moment orbital dû à la circulation des électrons et d'un moment de spin propre à l'électron; d'autre part, les champs électriques intracristallins perturbent très inégalement ces deux catégories de moments [I].

Moments atomiques dans les métaux. - Les effets gyromagnétiques montrent [2] que le moment des ferromagnétiques est principalement un moment de spin, mais le problème n'en reste pas moins compliqué, car le moment magnétique atomique dépend à la fois du partage numérique des électrons entre les étages $3 d$ et $4 s$, et de la structure interne de la couche $3 d$ pour un nombre total donné d'élec- trons. Les résultats spectroscopiques indiquent que l'atome libre de nickel, dans l'état le plus stable, possède 8 électrons dans l'étage $3 d$ et 2 électrons dans l'étage $4 \mathrm{~s}$, ce qu'on exprime par la notation abrégée $3 d^{8} 4 s^{2}$. Mais, il convient de noter que d'autres configurations fort différentes, telles que $3 d^{9} 4 s$ ou $3 d^{10}$ - dans cette dernière, il n'y a plus d'électrons $4 s-$ possèdent des énergies qui ne diffèrent que de moins d'un électron-volt de l'énergie de l'état fondamental.

Cette remarque tire son importance de ce qu'à l'état métallique, les énergies de liaison sont supérieures à un électron-volt, comme on peut s'en rendre compte d'après les données numériques sur les chaleurs de sublimation ou de vaporisation. On conçoit alors facilement que le passage des atomes libres à l'état métallique perturbe profondément la répartition numérique des électrons entre les étages $3 d$ et $4 s$ : on imagine même assez bien que l'équilibre soit dynamique, que les atomes d'un métal ne soient pas tous simultanément dans le même état et que le nombre moyen, par atome, des électrons \& $s$ soit fractionnaire. Ce problème difficile n'a pas reçu, jusqu'ici, de solutions satisfaisantes et je ne m'en occuperai pas davantage. Je supposerai simplement que le moment magnétique atomique d'un corps donné conserve une valeur invariable, indépendante de la température et de l'aimantation.

Propriétés magnétiques des éléments de transition. - Si tout se passait comme dans les sels, les propriétés magnétiques des métaux en question devraient être données, en gros, par la loi de Curie donnée en [I], mais il n'en est pas du tout ainsi et il faut, à cet égard, partager les éléments de transition en deux groupes [3] : le premier groupe est celui des métaux ferromagnétiques, fer, cobalt et riickel, pour lesquels un champ magnétique extérieur faible suffit, malgré l'agitation thermique, à aligner parallèlement les moments atomiques élémentaires. Le deuxième groupe comprend le manganèse et le chrome qui possèdent un paramagnétisme faible caractérisé par une aimantation proportionnelle au champ magnétique et indépendante de la température. Tous ces phénomènes doivent être attribués aux interactions entre les moments magnétiques élémentaires. 
Premier groupe : Ferromagnétiques.

Si les interactions entre atomes voisins tendent à aligner parallèlement les moments magnétiques, il se forme, d'après Weiss, des agrégats, des blocs d'atomes à moments parallèles qu'il a appelés des domaines élémentaires. Barkhausen le premier, et depuis beaucoup d'autres, ont mis en évidence expérimentalement l'existence de tels domaines. Le champ magnétique extérieur a simplement pour rôle d'aligner parallèlement l'aimantation des différents domaines.

M. Weiss a montré que les interactions étaient équivalentes à un champ magnétique fictif, le champ moléculaire, superposé au champ extérieur et proportionnel à l'aimantatior $I$

$$
H_{m}=n \mathfrak{J}
$$

$n$ est appelé le coefficient du champ moléculaire.

Ainsi, un ferromagnétique n'est qu'un paramagnétique dont l'aimantation est donnée par la loi [I], dans lequel règne un champ moléculaire donné par [2]. L'analyse montre qu'au-dessous d'une température donnée par $\Theta=n C_{0}$, le corps s'aimante spontanément dans un champ extérieur nul; il est ferromagnétique, $\Theta$ est le point de Curie. Aux températures supérieures à $\Theta$, le corps devient simplement paramagnétique avec [1/1]

$$
\mathscr{Y}=\frac{C_{0} H}{T-\Theta} \text {. }
$$

Différentes questions se posent maintenant que nous allons examiner.

Origine du champ moléculaire. - Après plusieurs tentatives infructueuses d'explication, on s'accorde maintenant, après Heisenberg, pour attribuer le champ moléculaire à des actions d'échange entre électrons [5] : ce sont d'ailleurs des actions électrostatiques auxquelles le principe d'exclusion de Pauli donne un caractère magnétique. Malheureusement, avec les moyens actuels de calcul, il est impossible d'obtenir une valeur approchée de $n$, ni même son signe. Ce sera donc une donnée purement expérimentale.

Légitimité de l'hypothèse du champ moléculaire. - Est-il correct de supposer que les interactions magnétiques sont simplement équivalentes à un champ moléculaire ? Remarquons d'abord que cette hypothèse n'est pas indispensable et qu'il est possible, notamment dans le cas d'une chaîne linéaire, de pousser jusqu'au bout les calculs sans introduire nulle part la notion de champ moléculaire. Mais il est exceptionnel que l'on puisse développer les calculs sans approximations et, en général, il faut simplifier et schématiser : c'est l'hypothèse du champ moléculaire qui conduit aux calculs les plus simples. Il se trouve également et fort heureusement qu'elle donne des résultats au moins aussi bons que les autres méthodes; de plus, c'est une expression commode et qui fait image.

En fait, on rend compte de la sorte des principaux faits expérimentaux : cependant, de minimes désaccords obligent à retoucher cette hypothèse trop schématique.

Les deux points de Curie. - Dans la théorie de Weiss, l'aimantation spontanée $\sigma$ disparaît (fig. 1, courbe en traits pleins) en $\Theta_{\mu}$, au point où la droite

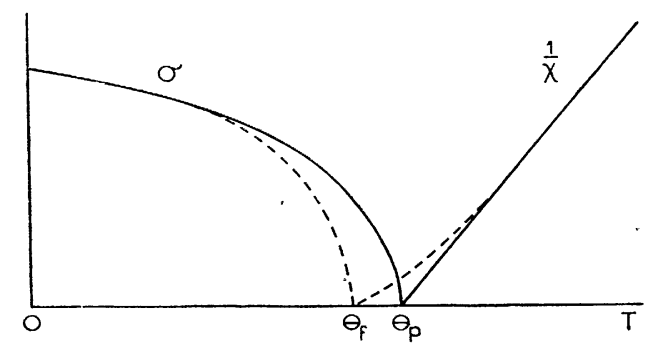

Fig. ı. - Les courbes en trait plein représentent schématiquement la variation thermique de l'aimantation spontanée et de l'inverse de la susceptibilité dans l'hypothèse du champ moléculaire simple. Les courbes en tirets montrent - les perturbations apportées par les fluctuations.

représentative des inverses des susceptibilités coupe l'axe des températures. En réalité, une courbure apparaît au voisinage de $\boldsymbol{\Theta}_{p}$, de sorte que la température réelle $\Theta$, de disparition de l'aimantation spontanée est inférieure à la température correspondant au point où la droite représentative extrapolée coupe l'axe des températures : on distingue ainsi le point de Curie paramagnétique $\Theta_{\rho}$ du point de Curie ferromagnétique $\Theta$. La différence entre les deux est de $20^{\circ}$ pour le nickel, de $1,5^{\circ}$ pour le fer.

J'ai pu [6], tout en conservant l'hypothèse du champ moléculaire, interpréter ces phénomènes en introduisant la notion de fluctuations du champ moléculaire. En effet, comme le champ moléculaire traduit des actions à très courte distance, les ondes d'agitation thermique provoquent des variations locales du champ moléculaire autour d'une valeur moyenne. Les calculs sont très pénibles : on ne peut préciser que sur des modèles très schématiques, ce qui ne change rien naturellement quant à la portée des résultats.

J'ai calculé [-], par exemple, l'influence des fluctuations sur la susceptibilité du nickel au-dessus du point de Curie (fig. 2). On voit que l'accord des valeurs calculées avec les valeurs expérimentales est excellent. Il est vrai qu'on disposait d'un paramètre de fluctuation qui a été choisi au mieux. Mais, la valeur de ce paramètre ainsi déterminée a été 
conservée dans les comparaisons suivantes qui prennent ainsi toute leur valeur.

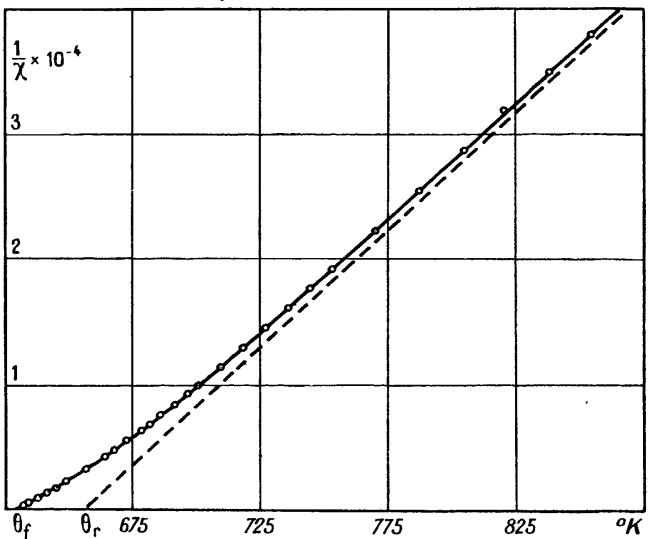

Fig. 2. - Inverse de la susceptibilité du nickel en fonction de la température. En traits pleins, la courbe calculée avec fluctuations, comparée aux valeurs observées indiquées par des cercles. La droite en tirets correspond au cas théorique sans fluctuations.

Aimantation spontanée au-dessus du point de Curie. - Les valeurs calculées [7] - aimantation spontanée au carré — ont été portées en trait plein sur la figure 3. Les valeurs expérimentales sont

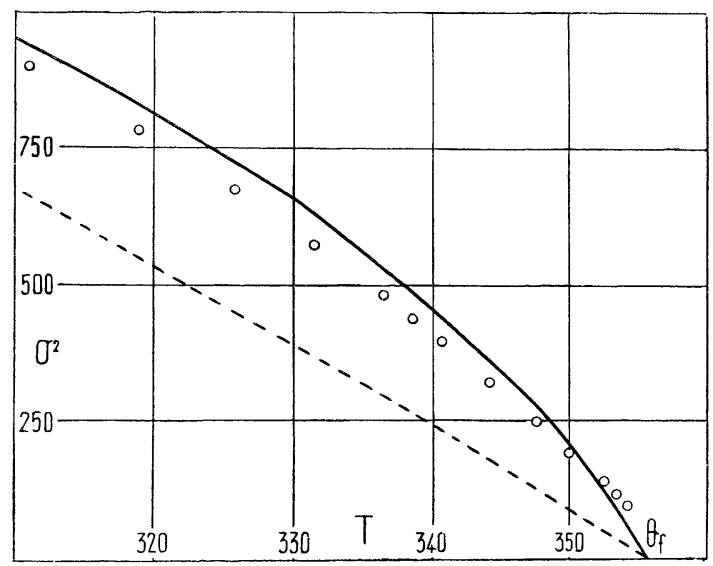

Fig. 3. - Aimantation spontanée du nickel dans la théorie classique sans fluctuations : courbe en tirets; avec les fluctuations : courbe en trait plein. Les données expérimentales (Weiss et Forrer) sont représentées par des cercles.

représentées par des cercles. L'accord réalisé est bien meilleur que celui que l'on obtiendrait avec l'hypothèse d'un champ moléculaire sans fluctuations. Les valeurs correspondantes ont été représentées en tirets sur la figure pour faciliter la comparaison.

Ghaleur spécifique. - Dans la théorie classique du champ moléculaire, la chaleur spécifique d'un ferromagnétique est anormale au-dessous du point de Curie, mais possède au-dessus la même valeưr

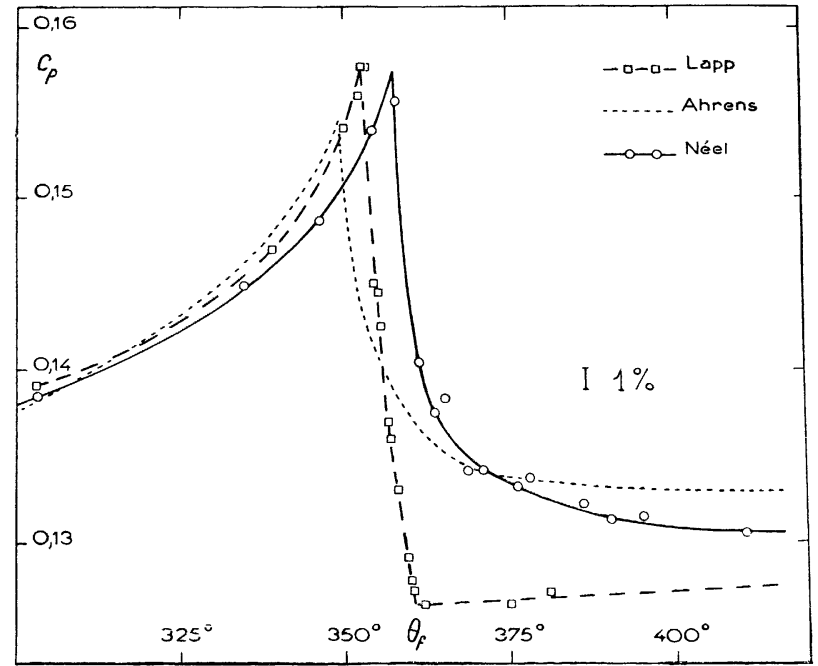

Fig. 4. - Données expérimentales sur la chaleur spécifique vraie du nickel, au voisinage du point de Curie, d'après les expériences de $\mathrm{M}^{\mathrm{m}} \mathrm{e}$ Lapp [8], d'Ahrens [9] et de Néel [10].

que celle d'une substance normale. Au point de Curie même, on devrait observer une discontinuité

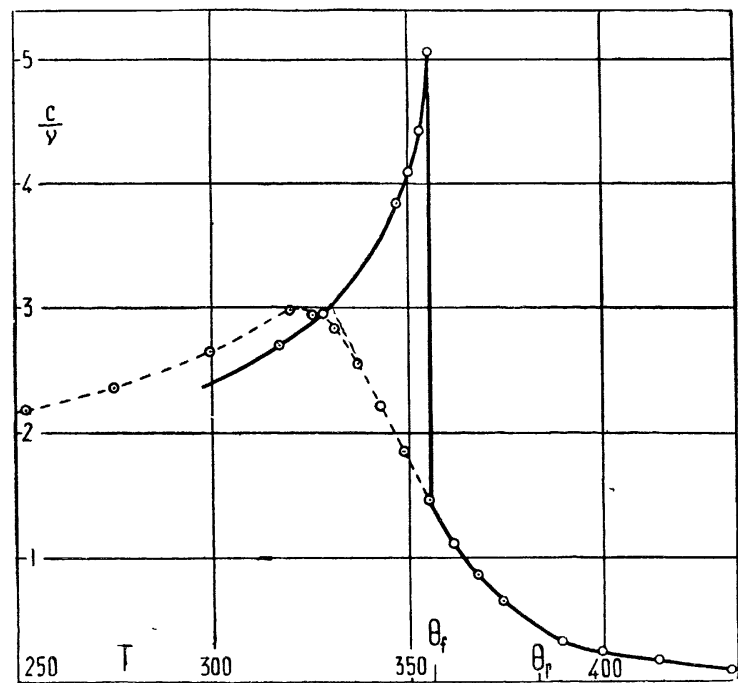

Fig. 5. - Influence des fluctuations sur la chaleur spécifique au voisinage du point de Curie. La courbe en trait plein donne le résultat du calcul quand on introduit des actions à longue distance égales au cinquantième seulement des actions à courte distance. La courbe en tirets est obtenue sans actions à longue distance. Au dessus de $\Theta$, les deux courbes se surerposent.

de la chaleur spécifique vraie. Or, l'expérience montre qu'il en est un peu autrement : les données les plus récentes (Ahrens, Néel, fig. १) montrent que l'anomalie de chaleur spécifique persiste encore 
à $50^{\circ}$ au-dessus du point de Curie et qu'il existe un arrondi commençant en $\Theta_{/}$. Ce phénomène montre qu'il doit subsister encore au-dessus de $\boldsymbol{\Theta}$, des amas très petits d'atomes à moments parallèles et les fluctuations doivent y jouer un rôle. Effectivement, le calcul fait exclusivement au moyen des données numériques obtenues plus haut [7] donne une chaleur spécifique représentée par la figure 5. Au-dessus du point de Curie, les résultats sont très sûrs. Au-dessous, la chaleur spécifique est très sensible à l'existence éventuelle d'un petit champ moléculaire à longue distance, tandis que la susceptibilité paramagnétique ne l'est pas, aussi est-il préférable pour comparer la théorie à l'expérience de se limiter aux températures supérieures à $\Theta_{/}$. C'est ce qui a été fait dans la figure 6 . La courbe calculée [II], en traits pleins, représente bien les points expérimentaux qui sont figurés par les cercles.

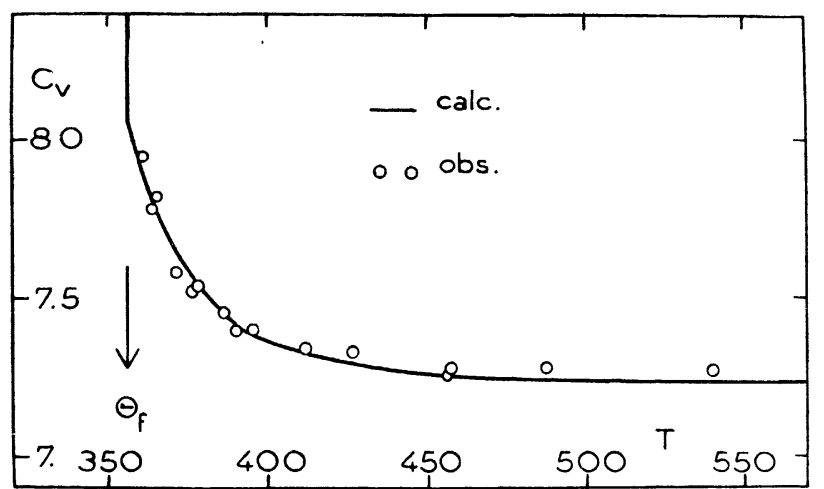

Fig. 6. - Comparaison des valeurs expérimentales (cercles) obtenues par Néel [10] aux valeurs calculées (courbe en trait plein) avec l'hypothèse des fluctuations du champ moléculaire.

Régions sensibles aux fluctuations du champ moléculaire. - Je conclurai cette discussion en disant que les retouches qu'apporte à la théorie classique l'introduction des fluctuations du champ moléculaire ne sont sensibles qu'au voisinage du point de Curie. Par exemple, pour le nickel, elles sont négligeables quand on se trouve à plus de I $_{0}{ }^{\circ}$ du point de Curie, vers le haut ou vers le bas. Il est donc prudent, si l'on s'en tient à des théories simplifiées, telles que la théorie classique du champ moléculaire, d'exclure dans les applications la région $\mathrm{du}$ point de Curie.

\section{Deuxième groufe : Antiferromagnétilues.}

Interprétons maintenant les propriétés magnétiques du deuxième groupe auquel appartiennent le manganèse et le chrome. On peut supposer ici que les interactions entre les moments élémentaires tendent à les disposer antiparallèlement ou, en adoptant le langage du champ moléculaire, supposer que le coefficient $n$ est négatif. L'application de la théorie de Weiss fait alors prévoir une variation thermique de la susceptibilité (3), avec la constante de Curie habituelle, qui est formellement la même que celle que l'on obtient avec $n$ positif, mais cette fois-ci $\Theta$ est négatif. Comme la susceptibilité du manganèse est sensiblement indépendante de la température, il faut abandonner la théorie ou, tout au moins, la perfectionner. Or, on montre qu'en tenant compte de phénomènes analogues aux fluctuations dans le cas de $n$ positif, la susceptibilité devient indépendante de la température, vers les basses températures, c'est-à-dire les températures qui sont petites à côté de la valeur absolue du point de Curie.

Susceptibilité des antiferromagnétiques à basse température [12]. - On conçoit la formation progressive vers le zéro absolu d'assemblages stables, à moments élémentaires magnétiques antiparallèles, qui se déforment ensuite sous l'action du champ magnétique et dont les déformations sont, en première approximation, indépendantes de la température.

Dès I 93 I, en utilisant un modèle classique, j'ai montré que la susceptibilité correspondante $\chi_{0}$ était donnée par la formule

$$
-2 n \chi_{0}=A,
$$

où $A$ est un coefficient qui dépend du réseau et qui est de l'ordre de grandeur de $\mathbf{I}$.

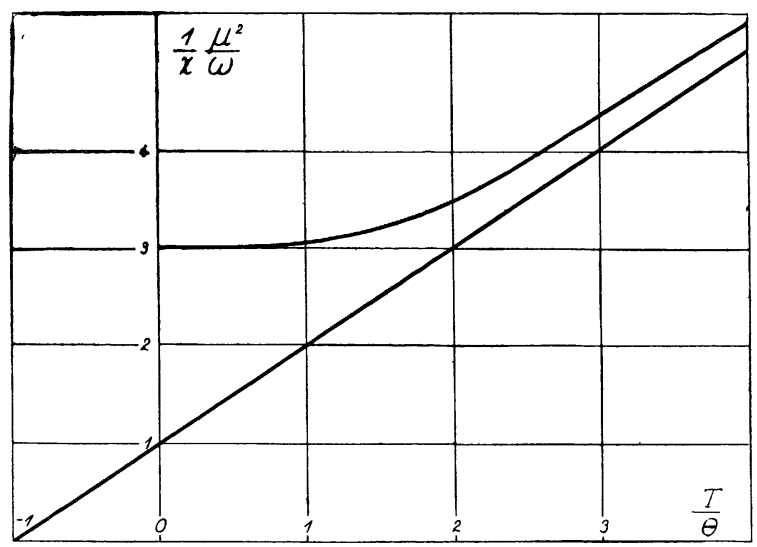

Fig. 7. - Courbe représentant la variation thermique de la susceptibilité d'une substance à champ moléculaire négatif. Les ordonnées sont proportionnelles aux inverses des susceptibilités. On a également tracé l'asymptote donnée par la loi de Curie-Weiss.

La susceptibilité s'éloigne de la valeur donnée par la formule (4) lorsque la température $T$ devient de l'ordre de grandeur de $+\boldsymbol{\Theta}$ en désignant - $\boldsymbol{\Theta}$ par le point de Curie de la formule de Curie-Weiss. L'ensemble de la variation thermique est représentée par une courbe telle que celle de la figure 7 . 
La droite qu'on y voit également tracée, asy mptote à la courbe en question, est donnée par la théorie classique du champ moléculaire. Il existe donc une grande différence entre la théorie classique et la théorie quantique, d'autant plus que, dans le cas du manganèse et du chrome, $\Theta$ est de l'ordre de grandeur de quelques milliers de gauss : à la température ordinaire, le point représentatif est donc nettement dans la partie horizontale de la courbe.

Ces conceptions sur le rôle et les effets du champ moléculaire négatif, d'abord accueillies avec quelques réticences par les théoriciens, sont maintenant généralement admises, et Bitter a appelé les substances correspondantes " antiferromagnétiques ". La théorie quantique rigoureuse est très difficile et ce n'est que récemment [r3] que Hulthèn, élève de Kramers, a traité le problème et notamment retrouvé la formule (4) avec un coefficient qui est toujours du même ordre de grandeur que celui que donne la théorie non quantique et qui lui est même identique dans des cas particuliers.

L'intérêt principal de la formule (4) est de permettre de calculer le coefficient $n$ des substances antiferromagnétiques à partir de la susceptibilité à basse température, indépendamment du moment atomique qui ne figure pas dans la formule.

Localisation des interactions magnétiques. - L'exposé précédent montre qu'une substance à moment atomique possède des propriétés extrêmement différentes suivant le signe de $n$, et il convient donc d'en étudier empiriquement les variations, car la théorie est sur ce point trop peu développée pour être très utile. Ia remarque suivante permet de simplifier cette étude : les interactions décroissent très rapidement quand la distance des atomes croît. Théoriquement, cette propriété découle du fait que les interactions sont liées à l'empiètement des fonctions d'onde électroniques des deux atomes, mais il existe aussi des preuves expérimentales plus directes : les sels halogénés des éléments de transition ont des températures de Curie assez voisines du zéro absolu, bien que les atomes de métal soient assez proches les uns des autres : c'est ainsi que dans le chlorure de nickel les atomes sont à $3,5 \AA$ les uns des autres. Cette distance est précisément égale à la distance de l'atome central à la deuxième couche de voisins dans le nickel métallique pur. Il est donc probable que les interactions magnétiques $\mathrm{du}$ nickel, pour leur plus grande part, ne sont dues qu'à la première couche de douze voisins. Les atomes plus éloignés n'ont qu'un rôle négligeable, et il en sera généralement ainsi sauf dans des cas exceptionnels.

On peut donc maintenant caractériser les interactions par un nouveau coefficient $u$, obtenu en divisant $n$ par le nombre $2 p$ des plus proches voisins. Le coefficient $u$ ne dépend plus que de la distance et de la nature des deux atomes voisins interagis- sant; il s'agit désormais d'étudier cette dépendance.

Relation entre l'interaction magnétique et la distance des atomes [14], [15]. - A la couche électronique $3 d$ correspond un nuage électronique de figure compliquée, mais qu'en première approximation on suppose de symétrie sphérique. On peut alors définir un rayon moyen de la couche magnétique dont l'emploi est commode dans certains problèmes. Ce rayon moyen peut être calculé par des formules semi-empiriques telles que celles de Slater [I6].

$A$ priori, le coefficient réduit $u$ du champ moléculaire doit au moins dépendre de deux variables : $r$, rayon moyen de la couche magnétique et $d$, distance entre les deux atomes interagissant comptée de centre à centre. Mais, il s'est finalement trouvé qu'en première approximation, le coefficient $u$ ne dépend, en réalité, que d'une seule variable : la plus courte distance entre les couches magnétiques, égale à la différence entre la distance $d$ des atomes et la somme $2 r$ des rayons de leur couche magnétique.

Cette propriété se vérifie aussi bien pour les ferromagnétiques que pour les antiferromagnétiques, comme le montre la figure 8 . Les interactions sont

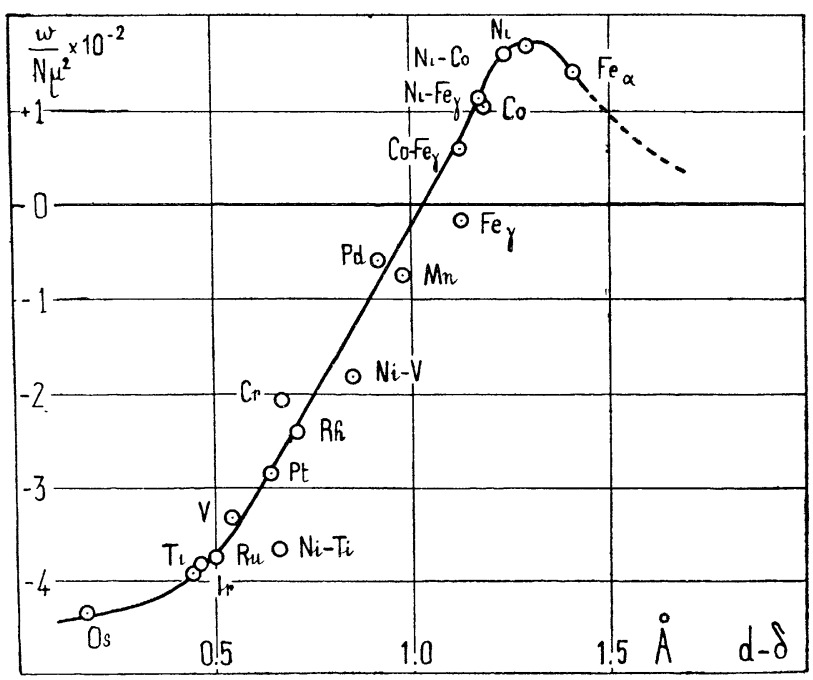

Fig. 8. - Interactions magnétiques en fonction de la distance des couches magnétiques. En ordonnée, on a porté $\omega: N \mu^{2}$ égal à $u$; en abscisse, $\mathrm{d}-\delta=\mathrm{d}-2 r$, plus courte distance entre les couches magnétiques.

d'abord fortement négatives (antiferromagnétisme) quand $d-2 r$ est petit, elles croissent ensuite algébriquement quand $d-2 r$ augmente, deviennent positives, on passe ainsi dans le domaine des substances ferromagnétiques, on atteint un maximum qui correspond à peu près à l'exemple du nickel pour $d-2 r=1,3 \AA$, puis les interactions diminuent avec la distance sans qu'on puisse préciser la loi de décroissance. Il est bien évident que, si 
l'on remonte à l'origine même des interactions, l'exactitude rigoureuse de la relation que l'on vient de mettre en évidence est bien peu vraisemblable : il s'agit plutôt seulement d'une relation approchée qui, néanmoins, rend de grands services.

Cas du manganèse [ $\mathrm{r} /$ i]. - C'est ainsi que les propriétés du manganèse sont facilement expliquées par la courbe de la figure 8. A l'état métallique, le point représentatif se trouve dans la région négative, à gauche. Une dilatation du réseau augmente $d$, donc $d-2 r$, et, si elle est suffisante, rend les interactions positives. L'introduction d'azote qui dilate le réseau rend, en effet, le manganèse ferromagnétique. Le ferromagnétisme d'un grand nombre de composés binaires de manganèse s'explique aussi par l'augmentation de la distance des atomes de manganèse, de même que le paramagnétisme, variable selon la loi de Curie-Weiss, des solutions solides étendues de manganèse dans le cuivre, l'argent ou l'or.

Terres rares $d u$ groupe yttrique à l'état métallique [I7]. - Une autre application intéressante est fournie par les métaux des terres rares pour lesquels, par suite d'une compensation entre le diamètre de la couche magnétique et la distance interatomique, $d-2 r$ est constant à 2 ou 3 centièmes d'angström près : $u$ est donc constant, $n$ est aussi constant, car le réseau est le même dans la série considérée et le point de Curie ne dépend plus que des nombres quantiques $L, S$ et $J$ par l'intermédiaire

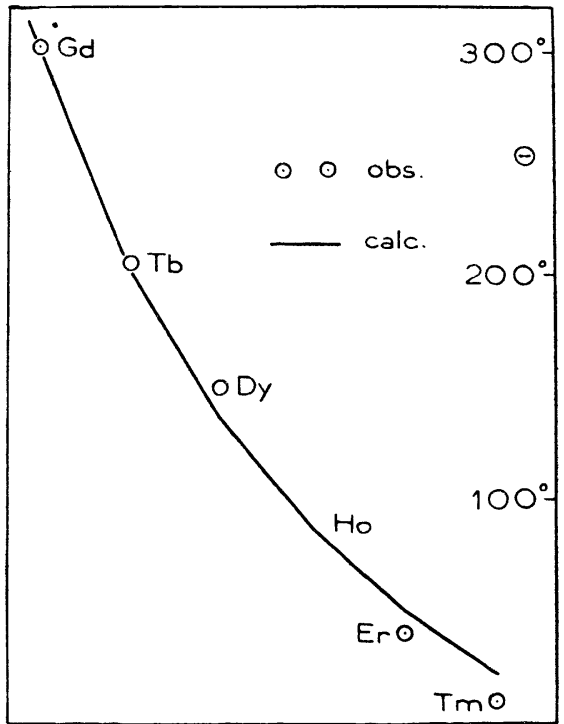

Fig. 9. - Points de Curie observés et points de Curie calculés pour les métaux du groupe yttrique. Les données expérimentales sont empruntées à Trombe et à Klem (cf. réf. [17]).

d'une formule très simple. On a représenté sur la figure 9 les points calculés et les points observés : on constate que l'accord est satisfaisant.
Anomalies de volume [15]. - La forme particulière de la courbe qui représente $u$ en fonction de la distance interatomique va nous permettre de comprendre les anomalies de volume et de dilatation que présentent les ferromagnétiques. Prenons d'abord une substance magnétique dont l'aimantation résultante est nulle, même quand il s'agit d'un domaine élémentaire : en un mot, une substance dépourvue d'aimantation spontanée (paramagnétique, ferromagnétique au-dessus du point de Curie) : deux atomes voisins quelconques sont en équilibre à une distance moyenne $d$ l'un de l'autre. Supposons maintenant qu'il apparaisse une aimantation spontanée $\sigma$, il s'introduit de ce fait une énergie magnétique

$$
W_{M}=-\frac{1}{2} n \sigma^{2}
$$

Si le point représentatif de $n$ se trouve dans la partie ascendante de la courbe d'énergie d'interaction (partie gauche de la figure 8), on s'aperçoit qu'une augmentation de la distance interatomique $d$ augmente algébriquement $n$, c'est-à-dire diminue

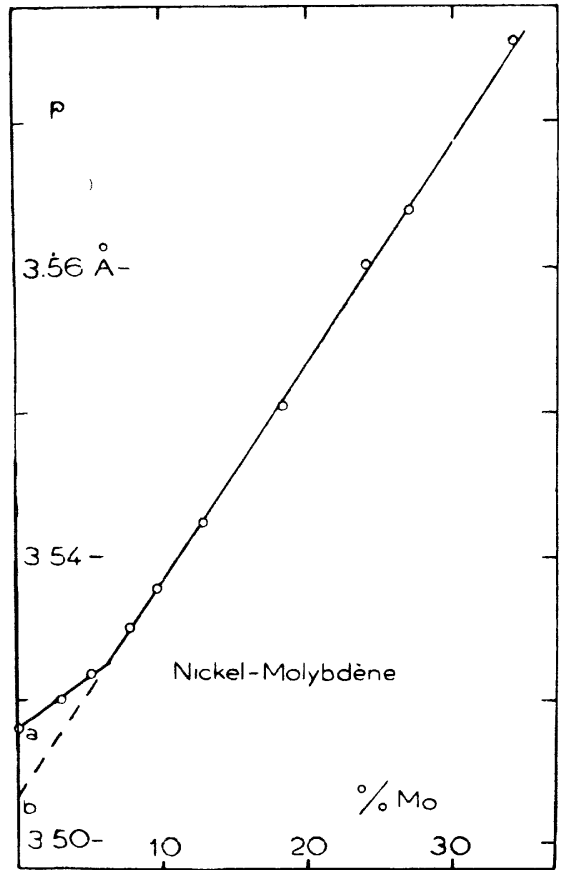

Fig. ı o. - Paramètre des solutions solides de molybdène dans le nickel. A gauche du coude, la solution solide est ferromagnétique à la température ordinaire. A droite du coude, les solutions sont à l'état paramagnétique.

l'énergie magnétique $W_{M}$ : l'apparition de l'aimantation spontanée doit donc être accompagnée d'une expansion de la substance limitée d'ailleurs par l'apparition simultanée d'une énergie élastique de 
déformation proportionnelle au carré de cette dernière. Inversement, si le point représentatif de $n$ se trouve sur la partie descendante de la courbe d'énergie d'interaction, l'apparition de l'aimantation spontanée est accompagnée d'une contraction de la substance.

Des expériences de Koster et Schmidt mettent en évidence ce phénomène. Prenons les solutions solides de molybdène dans le nickel. A la température ordinaire, elles sont ferromagnétiques jusqu'à 8 pour roo environ de molybdène. Lorsque la concentration de molybdène est supérieure, elles sont paramagnétiques, car le point de Curie est descendu au-dessous de la température ordinaire; de telles solutions solides ne possèdent donc pas d'aimantation spontanée et, si l'on porte sur un graphique le paramètre du réseau en fonction de la concentration, on obtient la droite représentée sur la partie droite de la figure io. On voit nettement qu'au-dessous de 8 pour ıoo, le paramètre du réseau est plus grand que celui que l'on obtient par extrapolation de la droite. En extrapolant jusqu'au nickel pur, on voit que le nickel, privé d'aimaritation spontanée, a un paramètre $b$ plias petit que le paramètre $a$ du nickel normal ( $b=3,505$ et $a=3,5$ เ $5 \AA$ ).

Le même phénomène se produit dans les solutions solides de cobalt-molybdène, mais beaucoup plus accentué.

Anomalies de dilatation des ferromagnétiques [14]. — Pour préciser les considérations ainsi esquissées, il faut tenir compte de la dilatation

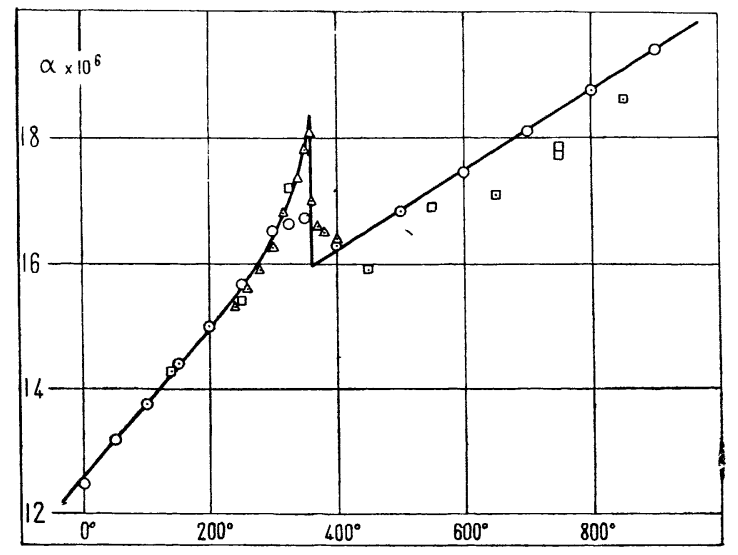

Fig. ı ı. - Coefficient de dilatation vrai du nickel calculé (courbe en trait plein) comparé aux points expérimentaux : $\odot$ Chevenard [18], [.] Hidnert [19], $\triangle$ Williams [20].

thermique et, dans ces conditions, on trouve que l'anomalie de longueur $\delta l$, c'est-à-dire la différence entre la longueur réelle de la substance et celle qu'elle aurait si l'on pouvait la priver de son aimantation spontanée, est donnée par la formule

$$
\frac{\partial l}{l}=p \sigma^{2}\left[\frac{\mathrm{I}}{2-A d} u^{\prime}+\alpha T\left(\frac{u^{\prime \prime}}{A}-\frac{u^{\prime \prime \prime}}{6 B}\right)\right],
$$

où $T$ est la température absolue, $\alpha$ le coefficient de dilatation; $A$ et $B$ sont deux coefficients qui dépendent des propriétés élastiques. Dans la formule (5) figurent $u^{\prime}, u^{\prime \prime}, u^{\prime \prime \prime}$ dérivées successives de l'énergie d'interaction $u$ par rapport à la distance interatomique. Ces dérivées sont les mêmes que les dérivées par rapport à $d-2 r$. M. Chevenard, à la suite des belles et nombreuses recherches qu'il a entreprises avec ses différents modèles de dilatomètres enregistreurs, avait trouvé que l'anomalie de dilatation était représentée par une formule du type

$$
\frac{\partial l}{l}=\sigma^{2}\left(\lambda+\mu T+\nu^{2} T^{2}\right),
$$

comme précisément la formule (5).

Cette formule représente d'ailleurs les expériences avec une bonne précision, comme le montrent les figures i i et i 2 sur lesquelles on peut comparer les points expérimentaux, obtenus par Chevenard et d'autres auteurs, à la courbe de dilatation déduite de la formule (6) avec des coefficients convenables.

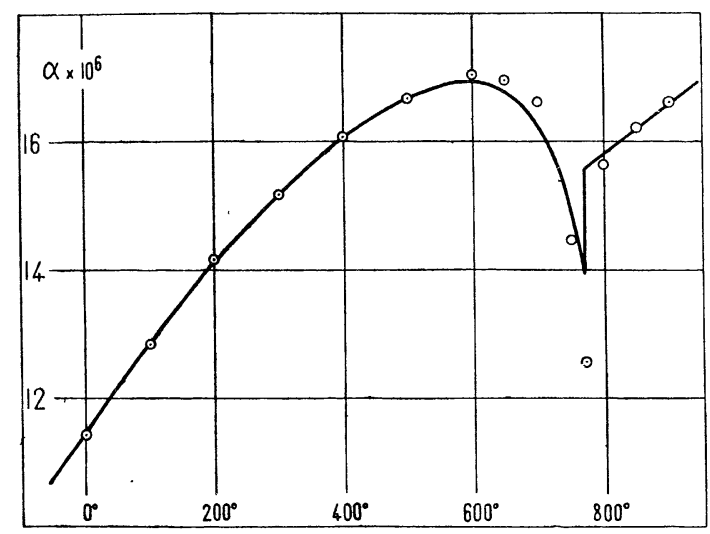

Fig. I 2. - Coefficient de dilatation vrai du fer calculé (courbe en trait plein) comparé aux points expérimentaux $\odot$ de Chevenard [18].

Les coefficients de la formule (6) une fois connus de cette manière, il suffit de les comparer aux coefficients de la formule (5) pour en déduire $u$ (ou ce qui revient au même $n$ ) et ses dérivées successives $u^{\prime}, u^{\prime \prime}, u^{\prime \prime \prime}$, par rapport à la distance. A cet égard, la théorie présente un grand intérêt : en effet, connaissant $u, u^{\prime}, u^{\prime \prime}, u^{\prime \prime \prime}, \ldots$, on peut, au moyen d'un développement en série de Taylor, restituer la forme de la courbe $u=f(d-2 r)$ au voisinage $\mathrm{du}$ point représentatif du métal en question.

La méthode a été appliquée au nickel : la figure s 3 en illustre les résultats. La courbe en trait plein 
provient d'un développement en série dont les coefficients ont été calculés par la formule (5) appliquée aux résultats expérimentaux de Chevenard [18] relatifs au nickel. Les cercles proviennent du calcul direct de $u$ au moyen du point de Curie des substances indiquées. On voit facilement que la courbe a la même allure générale que la courbe de la figure 8 et que, bien mieux, elle représente parfaitement les points expérimentaux déduits de l'étude directe du cobalt et de différentes séries d'alliages par une méthode entièrement différente.

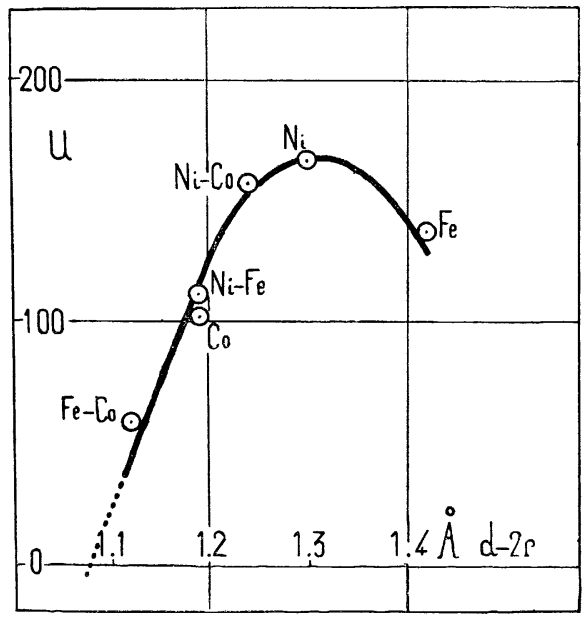

Fig. I 3. - Courbe donnant le coefficient du champ moléculaire en fonction de la distance des couches magnétiques, calculé d'après les anomalies de dilatation du nickel.

Variation thermique du champ moléculaire [14]. - Les exemples précédents précisent suffisamment la dépendance du champ moléculaire et des distances interatomiques pour qu'on puisse aborder le problème de la variation thermique du champ moléculaire, car, à cet égard, nous avions jusqu'ici supposé les atomes immobiles aux nœuds d'un réseau indéformable.

L'effet de la température est double : en premier lieu, il se produit une dilatation générale du réseau qui a pour effet d'augmenter également toutes les distances interatomiques entre atomes voisiris, d'où une variation de $n$ qui se déduit de la courbe de la figure 8. En second lieu, il se produit, par élévation de température, des oscillations de plus en plus amples des atomes autour de leur position moyenne d'équilibre; si le coefficient du champ moléculaire était une fonction linéaire de la distance, les variations périodiques de $n$, par agitation thermique, n'altéreraient pas sa valeur moyenne, mais comme il existe une courbure, il y a là une nouvelle cause de variation thermique de $n$.

Finalement, on trouve que $n$ est une fonction de la température qui se développe sous la forme suivante

$$
n=n_{0}\left(\mathrm{I}+l T+m T^{2}+\ldots\right) ;
$$

le coefficient du terme en $T$ est d'ailleurs [1 4] égal à

$$
l=\frac{\alpha d}{u} u^{\prime}+\frac{R \varkappa_{0} d^{2}}{6 V u} u^{\prime \prime} ;
$$

$R$ est la constante des gaz, $x_{0}$ le coefficient de compressibilité du métal, $V$ son volume atomique. Le premier et le second terme de la formule (8) correspondent respectivement au premier et au second effet que je viens de signaler. Pratiquement, jusqu'à $1000^{\circ}$, on peut négliger le terme en $T^{2}$ de la formule (7), mais pas du tout le terme en $T$ qui, à cette température, peut dépasser 20 pour roo du terme indépendant de la température.

Cette variation thermique du champ moléculaire a une assez grande importance théorique; en effet, l'aimantation d'un paramagnétique pur est donnée par la loi de Curie (I) où $C_{0}$ est la constante de Curie liée au moment atomique par les formules connues. Mais, lorsqu'il existe un champ moléculaire à variation thermique, l'aimantation est donnée par une formule telle que la formule (3) qui a bien la même forme que la loi de Curie-Weiss, mais dans laquelle la constante de Curie $C_{0}$ est remplacée par la constante $C$ qui est liée à la constante de Curie $C_{0}$ du paramagnétisme pur par la relation

$$
C=(\mathrm{I}+l \Theta) C_{0} \text {. }
$$

C'est ainsi que dans le nickel on a : $C=0,323$ et $C_{0}=0,379$. Il est bien évident que l'expérience donne directement $C$, mais cette quantité, telle quelle, ne nous intéresse pas : il faut en déduire $C_{\mathbf{0}}$ et, pour cela, connaître le coefficient du binome de variation thermique du champ moléculaire. On voit ici les conséquences inattendues de la variation thermique du champ moléculaire avec la distance des atomes.

Notons encore que, dans la région ferromagnétique, cette variation thermique de $n$ provoque des modifications sensibles et auxquelles il faut avoir égard de la variation thermique de l'aimantation spontanée.

D'ailleurs, et d'un point de vue plus général, la prise en considération de ces phénomènes est de nature à modifier certaines conclusions qui avaient été adoptées relativement au moment élémentaire du nickel.

Conclusion. - On vient d'examiner dans ce qui précède, d'une manière assez approfondie, le côté " champ moléculaire » de la théorie du ferromagnétisme et, dans ce but, nous avions émis préalablement l'hypothèse d'un moment atomique invariable, indépendamment notamment de la température et du champ moléculaire. Cette hypothèse a été adoptée pour simplifier les calculs et l'exposé. Jusqu'ici, 
et malgré d'importants travaux, l'étude de l'aspect " moment élémentaire " du ferromagnétisme est beaucoup moins avancée. Il est certain que l'hypothèse la plus simple qui consiste à attribuer le même moment élémentaire à tous les atomes du métal est inexacte, puisque le moment atomique du nickel, exprimé en magnétons de Bohr, est fractionnaire. Il s'agit plutôt d'un équilibre entre atomes à moments différents dont les conditions exprimées dans un autre langage ont été précisées d'abord par Slater et Mott. En principe, cet équilibre est déplacé sous l'influence du champ moléculaire.

Un nouveau champ de recherche, peu exploré, s'ouvre ainsi devant nous. Il faut espérer que, malgré l'extrême complication des phénomènes, leur étude conduira à des résultats positifs.

Manuscrit reçu le i 5 décembre 1939 .

\section{BIBLIOGRAPHIE.}

[1] Sur ces questions, consulter le rapport très complet et très documenté de Van Vleck à la Réunion d'étude sur le Magnétisme de Strasbourg, en 1939 (à paraître prochainement).

[2] Voir le Rapport de Weiss au Conseil Solvay de r93o (Gauthier-Villars, Paris) et le Rapport de Barnett à la réunion de Strasbourg (Cf. réf. I).

[3] Pour être complet, rappelons que le palladium et le platine, qui appartiennent respectivement à la deuxième et à la troisième série de transition, suivent la loi de Curie avec un point de Curie négatif très grand et forment ainsi un troisième groupe, mais dont les propriétés se rattachent à celles du deuxième groupe.

[4] Au sujet de la théorie du champ moléculaire, consulter : Weiss, Le magnétisme (A. Colin, Paris).

[5] Pour des références détaillées aux Mémoires originaux, consulter les deux Ouvrages : VAN Vleck, Electric and Magnetic susceptibilities (Clarendon Press, Oxford, 1932) et Motт et Jones, Properties of Metals and Alloys (Clarendon Press, Oxford, i 936 ).

[6] L. Néel, Ann. de Physique, 1932, 17, p. 5.
[7] L. NÉEL, J. de Physique, 1934, 5, p. 104.

[8] E. LAPP, Ann. de Physique, r929, 12, p. 442.

[9] Ahrens, Ann. der Physik, i 934,21, p. 69.

[ı] L. Néel, C. R. Acad. Sc., r 938,207 , p. 1384.

[i I] L. Néel, C. R. Acad. Sc., i 938,208 , p. I 77.

[12] L. Néel, Ann. de Physique, 1932, 17, p. 5 et ig36, 5, p. 232 ; C. R. Acad. Sc., 1936, 203, p. 104.

[13] L. Hulthén, Proc. Roy. Acad. Amsterdam, i936, 39, p. r9o; Arkiv f. Mat. Astr. o. Fysik, 1937, 26, no 11, p. 237 .

[14] L. Néel, Ann. de Physique, r937, 8, p. 237.

[15] Pour cette question et celles qui sont traitées dans les paragraphes suivants, consulter le Rapport de l'auteur à la Réunion d'étude sur le Magnétisme de Strasbourg en ${ }_{939}$ (à paraître prochainement).

[16] Slater, Phys. Rev,, i 930,36, p. 57.

[17] L. Néel, C. R. Acad. Sc., 1938, 206, p. 57; Z.f. Elektrochemie, 1939, 15, p. 378 .

[ 18$]$ P. Chevenard, Travaux et Mémoires du B. I. des Poids et Mesures, 17, Paris.

[19] Hidnert, Bur. of Standards, r93o, 5, p. г 305 .

[20] Williams, Phys. Rev., 1934, 46, p. гогі. 